\title{
Model-based Filter Design for Triple Skyhook Control of In-Wheel Motor Vehicles for Ride Comfort
}

\author{
Tomonori Suzuki $^{* a)}$ Student Member, Masahiro Mae* Student Member \\ Takuma Takeuchi* $^{* *}$ Non-member, Hiroshi Fujimoto* Senior Member \\ Etsuo Katsuyama** Non-member
}

(Manuscript received May 7, 2020, revised Dec. 11, 2020)

J-STAGE Advance published date : Feb. 19, 2021

\begin{abstract}
Vibration suppression control in vehicles is important for ride comfort. In vehicle electrification, the use of an in-wheel motor as an actuator for vibration suppression has attracted attention as an alternative to active suspension systems. However, a model-free conventional controller design method cannot sufficiently suppress vibration at the most significant frequencies $(4 \mathrm{~Hz}$ to $8 \mathrm{~Hz})$ because of mechanical resonance. In this study, we analyze the frequency response of an experimental vehicle. Using the identified frequency response of the vehicle, we design a pre-compensation filter based on the desired vibration suppression performance characteristics. The vibration suppression performance improved with the experimental vehicle at target frequencies from $4 \mathrm{~Hz}$ to $8 \mathrm{~Hz}$.
\end{abstract}

Keywords: vibration suppression control, vehicle motion control, electric vehicle, in-wheel motor, model-based design, ride comfort

\section{Introduction}

In recent years, electric vehicles (EVs) have attracted much attention as a result of increasing environmental awareness. EVs have high motion performance because of their motor characteristics. Electric motors have three significant advantages over internal combustion engines: their torque response is several hundred times faster and more accurate than that of the internal combustion engine, their output torque can be directly measured from the motor current value, and distributed small motors enable flexible arrangements ${ }^{(1)}$. By utilizing these features effectively, advanced vehicle motion control becomes possible ${ }^{(2)(3)}$.

In particular, the distributed arrangement of motors enables various drivetrain configurations in EVs. One such configuration is an in-wheel motor (IWM) system in which motors are mounted in each wheel. The IWM system has the advantages of reducing the total weight of a drivetrain and increasing the freedom of interior layout design ${ }^{(4)}$. From a control perspective, torque-vectoring enables higher motion performance, and the shorter driveshaft removes low-frequency resonances which create a substantial limitation in $\operatorname{control}^{(5)(6)}$.

To improve motion performance and ride comfort, several vibration suppression control methods that lie in several $\mathrm{Hz}$ bands have been proposed ${ }^{(7)-9)}$. However, the IWM system increases the unsprung weight and causes deterioration of ride comfort over $4 \mathrm{~Hz}^{(10)(11)}$. To solve this problem, studies

a) Correspondence to: Tomonori Suzuki. E-mail: tomonorisuzuki @ieee.org

* The University of Tokyo

5-1-5, Kashiwanoha, Kashiwa, Chiba 277-8561, Japan

** Toyota Motor Corporation

1200, Mishuku, Susono, Shizuoka 410-1193, Japan

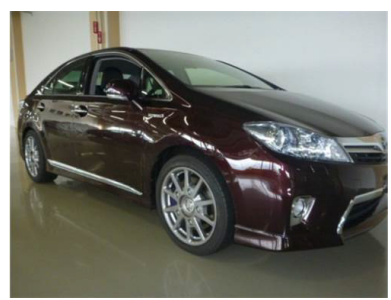

(a) Experimental vehicle.

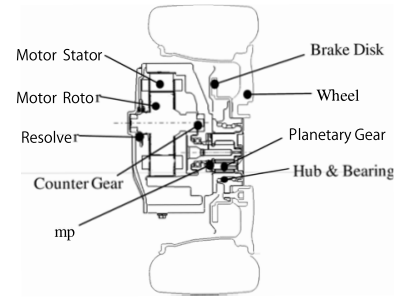

(b) IWM unit.
Fig. 1. Experimental vehicle with IWM units

on vibration suppression for the unsprung vertical axis mode and the longitudinal axis mode that lay in a higher frequency band (10 Hz and above) have been conducted ${ }^{(12)-(14)}$.

These vibration suppression control methods at high bandwidths use the high torque responsiveness of the IWM system. As described above, extensive research using the IWM system has been carried out to suppress vibration in a wide frequency range ${ }^{(15)}$.

Katsuyama et al. proposed triple skyhook (tSH) control, which is a vertical vibration suppression control method ${ }^{(16)}$. This method has the potential of suppressing an extensive range of frequency vibration but uses a lowpass filter (LPF) with a low cutoff frequency to stabilize the feedback control system. The low-cutoff-frequency LPF limits the gain and delays the phase of the target frequency $4 \mathrm{~Hz}$ to $8 \mathrm{~Hz}$ and worsens the vibration suppression performance. Therefore, it is desired to stabilize the control system without using a low-cutoff-frequency LPF.

In this paper, we analyze vehicle vibration using a quartercar model and identify the frequency response of an experimental vehicle, which is described in section 2. In section 3, we reveal the problem of the conventional method. In section 


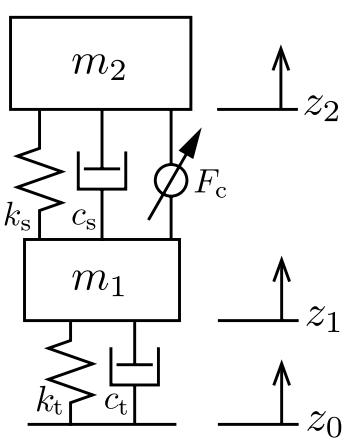

Fig. 2. Quarter-car model

Table 1. Vehicle model parameter definitions

\begin{tabular}{clcl}
\hline Symbol Meaning & \multicolumn{2}{c}{ Symbol Meaning } \\
\hline$m_{1}$ & Quarter unsprung mass & $m_{2}$ & Quarter sprung mass \\
$k_{\mathrm{s}}$ & Spring stiffness & $c_{\mathrm{S}}$ & Damping coefficient \\
$k_{\mathrm{t}}$ & Tire stiffness & $c_{\mathrm{t}}$ & Tire damping coefficient \\
$z_{0}$ & Road displacement & $z_{1}$ & Unsprung mass displacement \\
$z_{2}$ & Sprung mass displacement & $F_{\mathrm{d}}$ & Driving force \\
$\theta$ & Angle of instant suspension rotation & $F_{\mathrm{c}}$ & Suspension reaction force \\
$r$ & Radius of tire & $T$ & Motor torque \\
\hline
\end{tabular}

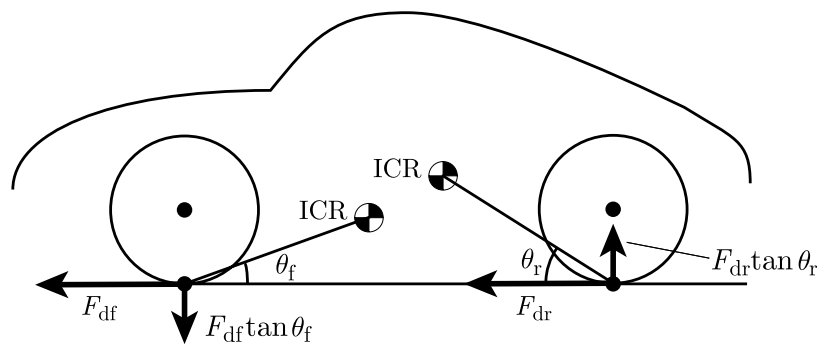

Fig. 3. Suspension reaction force

4, we propose the design method of a pre-compensation filter for suppressing the mechanical resonance excitation, which causes instability, and improving the vibration suppression performance. We can design the pre-compensation filter with desired frequency characteristics using a sensitive function. As an example, we designed the pre-compensation filter to suppress the vibration at $4 \mathrm{~Hz}$ to $8 \mathrm{~Hz}$, which is most significant for drivers ${ }^{(17)}$. In section 5, we conducted experiments and validated the effectiveness of the proposed model-based filter design method.

\section{Model and Experimental Vehicle Analysis}

2.1 Experimental Vehicle with IWM We use the experimental vehicle shown in Fig. 1(a). The experimental vehicle has four IWM units shown in Fig. 1(b) and is equipped with four vertical acceleration sensors on the sprung mass above each wheel. The rear left part of the experimental vehicle is analyzed as a quarter-car model described in the following sections.

2.2 Vehicle Vibration Model The vehicle vibration is analyzed by the quarter-car model as shown in Fig. 2. This model consists of a quarter sprung mass, a quarter unsprung mass, suspension, and a tire. The definition of each variable is shown in Table $1 . F_{\mathrm{c}}$ is a suspension reaction force, which is described in section 2.3 and Fig. 3.

The motion equations of the quarter-car model are given as follows:
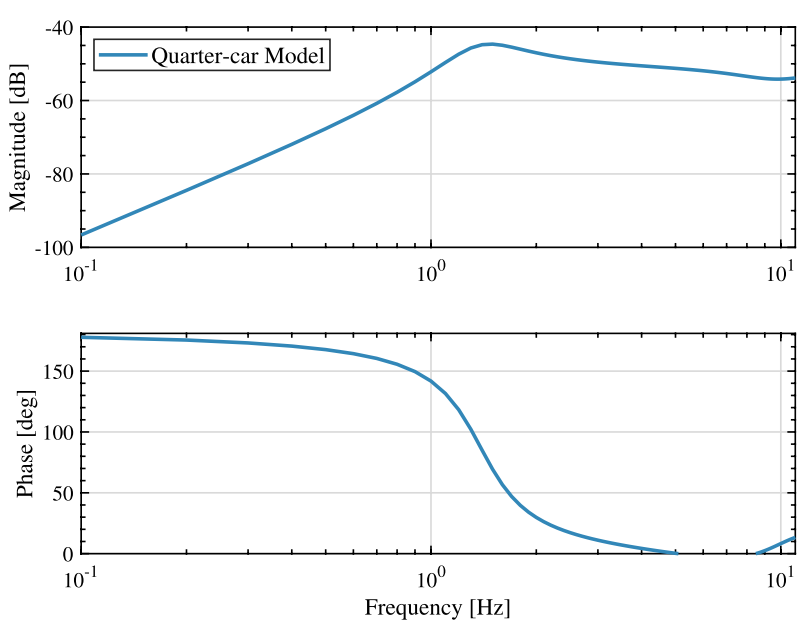

Fig. 4. Frequency response of the quarter-car model
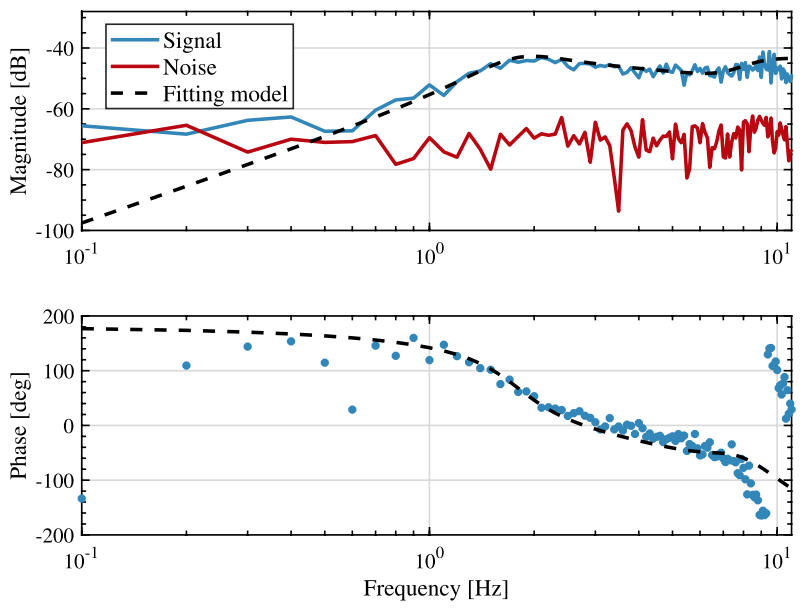

Fig. 5. Frequency response from motor torque to acceleration of sprung mass

$$
\begin{aligned}
& m_{2} z_{2} s^{2}=\left(c_{\mathrm{s}} s+k_{\mathrm{s}}\right)\left(z_{1}-z_{2}\right)+F_{\mathrm{c}}, \cdots \ldots \ldots \ldots \ldots \ldots \ldots \ldots \\
& m_{1} z_{1} s^{2}=-\left(c_{\mathrm{s}} s+k_{\mathrm{s}}\right)\left(z_{1}-z_{2}\right)+\left(c_{\mathrm{t}} s+k_{\mathrm{t}}\right)\left(z_{0}-z_{1}\right)-F_{\mathrm{c}}
\end{aligned}
$$

The transfer function model from motor torque to sprung mass acceleration of the quarter-car model is given as shown in (3), and its Bode diagram is shown in Fig. 4.

Using general passenger vehicle parameters, this transfer function has two resonances around $1.3 \mathrm{~Hz}$ and $10 \mathrm{~Hz}$ and one anti-resonance around $9.9 \mathrm{~Hz}$.

We use the grey box modeling in the system identification. From the result of the quarter-car model analysis, the frequency response of the experimental vehicle can be fitted to the sum of two resonance modes.

The first resonance comes from sprung mass and suspension stiffness. The second resonance comes from unsprung mass and tire stiffness. When the passengers' weight changes, the first resonance frequency changes slightly. However, the second resonance frequency does not change.

Though details will be described in section 4 , the second resonances cause instability in the feedback control system. The resonance which is suppressed by the filter is unaffected against passengers' weight change.

2.3 Suspension Reaction Force The suspension reaction force $F_{\mathrm{c}}$ is defined as the vertical element of 


$$
\begin{aligned}
& \frac{\ddot{z_{2}}}{F_{c}}=\frac{s^{2}\left(m_{1} s^{2}+c_{t} s+k_{t}\right)}{m_{1} m_{2} s^{4}+\left\{\left(m_{1}+m_{2}\right) c_{s}+m_{2} c_{t}\right\} s^{3}+\left\{\left(m_{1}+m_{2}\right) k_{s}+m_{2} k_{t}+c_{s} c_{t}\right\} s^{2}+\left(c_{s} k_{t}+c_{t} k_{s}\right) s+k_{s} k_{t}} \\
& P(s)=6 \times 10^{-3} s^{2}\left(\frac{0.7}{s^{2}+6.786 s+127.9}+\frac{0.3}{s^{2}+32.04 s+2852}\right) e^{-35 \times 10^{-3} s} \ldots \ldots \ldots \ldots \ldots \ldots \ldots
\end{aligned}
$$

suspension internal forces caused by the driving force.

Fig. 3 shows the lateral view of the simplified kinematic model of a vehicle with suspension reaction forces. The arm between the sprung and unsprung masses has a structure that rotates around the instant center of rotation (ICR). The driving force working on the tire in the longitudinal direction causes a moment in the arm about the ICR. The vertical force that balances the moment of the driving force is then generated.

Assuming that the angle between the ground and ICR of the arm is $\theta$, the magnitude of the driving force is $F_{\mathrm{d}}$, the slip ratio is a constant, and the suspension reaction force $F_{\mathrm{c}}$ in the vertical direction can be calculated as follows:

$$
F_{\mathrm{c}}=F_{\mathrm{d}} \tan \theta=r T \tan \theta .
$$

2.4 System Identification of Experimental Vehicle with IWM We identify the frequency response from the rear left wheel motor torque to the corresponding sprung vertical acceleration using a multi-sine input ${ }^{(18)}$. When the multisine input is applied to the rear left wheel motor, the remaining wheels' motor torques are set to 0 . The identified frequency range is from $0.1 \mathrm{~Hz}$ to $10 \mathrm{~Hz}$, which is the torque control bandwidth of the experimental vehicle. The identified frequency response and its fitting line are displayed in Fig. 5. This frequency characteristic has two resonances and one anti-resonance. The characteristic is the same as the quarter-car model. The frequency response function (FRF) is fitted using deadtime and the sum of several modes. The deadtime is estimated as $35 \mathrm{~ms}$, and the resonance frequencies are estimated as 1.8 and $8.5 \mathrm{~Hz}$. The total FRF $P(s)$ is expressed in equation (4).

\section{Vibration Suppression Control}

3.1 Triple Skyhook Control The tSH control feeds back the vertical displacement, velocity, and acceleration of the sprung mass ${ }^{(16)}$.

The transfer function of the quarter-car model from $z_{1}$ to $z_{2}$ is shown as follows:

$$
z_{2}=\frac{c_{s} s+k_{s}}{m_{2} s^{2}+c_{s} s+k_{s}} z_{1}
$$

Since only the second derivative of the sprung mass displacement $\ddot{z}_{2}$ is measured, the control input is calculated from that.

$$
F_{\mathrm{c}}=-\alpha \frac{m_{2} s^{2}+c_{s} s+k_{s}}{s^{2}} \ddot{z}_{2} D(s)
$$

where $\alpha$ is the control gain, and $D(s)$ is a pre-compensation filter. The pre-compensation filter consists of LPF for stabilization and HPF for eliminating integrated drift and road surface gradient effect in the conventional method. The lowcutoff-frequency LPF worsens vibration suppression performance. In this paper, we do not use an HPF to make it easier

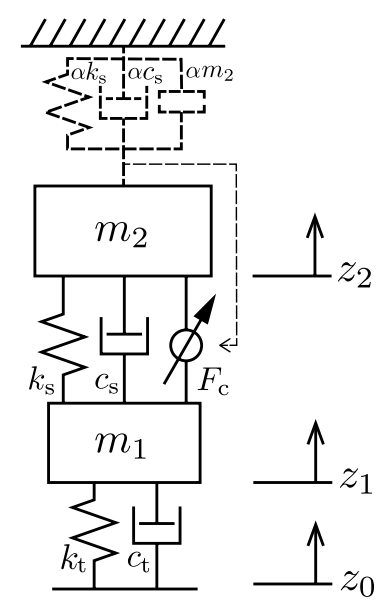

Fig. 6. Concept of triple skyhook control (Dotted lines express virtual elements of the control.)

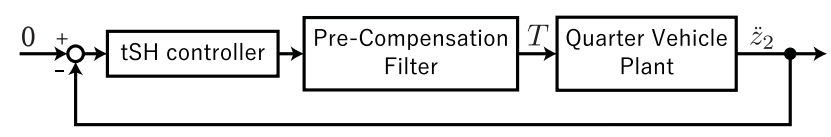

Fig. 7. Overall feedback control system block diagram

to compare the filter effect corresponds to the LPF. An HPF does not change the gain or phase over its bandwidth and does not affect LPF equivalent pre-compensation filter design. We describe the conventional and proposed pre-compensation filter in section 3.2 and 4.1 in detail.

The transfer function of the quarter-car model from $z_{1}$ to $z_{2}$ with this input is expressed as follows:

$$
z_{2}=\frac{c_{s} s+k_{s}}{\left(m_{2}+\alpha_{2} D(s)\right) s^{2}+\left(c_{s}+\alpha_{1} D(s)\right) s+\left(k_{s}+\alpha_{0} D(s)\right)} z_{1} .
$$

The control input can change the three parameters of the denominator in equation (8). When $\alpha_{2}=\alpha m_{2}, \alpha_{1}=$ $\alpha c_{s}, \alpha_{0}=\alpha k_{s}$, the tSH control method multiplies $z_{2}$ by $1 /(1+\alpha D(s))$ compared to the model without control. Physically, this means that the sprung mass is hooked with three virtual elements: spring, damper, and inerter ${ }^{(19)}$, as shown in Fig. 6.

The overall feedback control system block diagram is displayed in Fig. 7. tSH control with a pre-compensation filter $D(s)$ is applied to the plant.

3.2 Conventional Model-free Filter Stabilization If we apply the $\mathrm{tSH}$ control without any pre-compensation filter to the vehicle plant, the controller excites the mechanical resonance. The conventional tSH control uses a low cutoff frequency such as $3 \mathrm{~Hz} \mathrm{LPF}$ as a filter $D(s)$ to suppress the mechanical resonance. However, a low cutoff frequency LPF delays the phase at $4 \mathrm{~Hz}$ to $8 \mathrm{~Hz}$. Fig. 8 shows the Nyquist plots of the identified plants with tSH control with LPF. In Nyquist plots, the sensitive function corresponds to the 


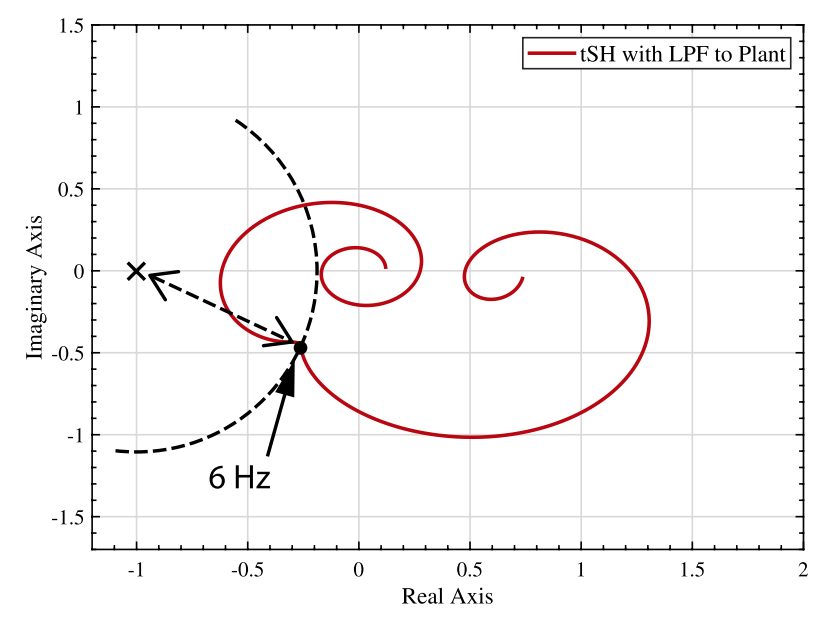

Fig. 8. Nyquist plots of plants with tSH control with LPF

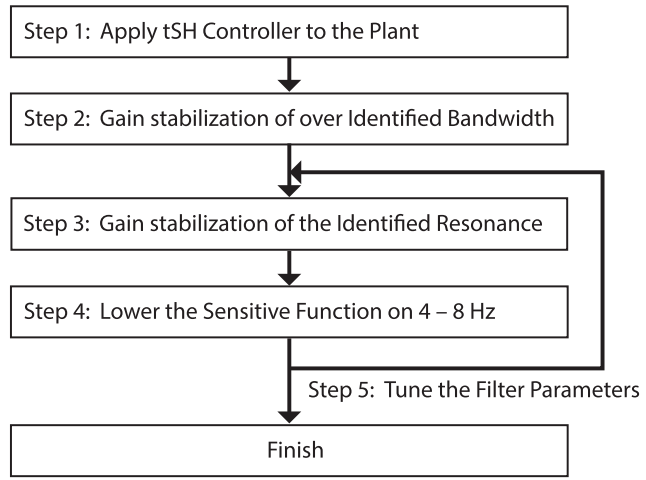

Fig. 9. Filter design procedure

inverse of the distance between the Nyquist plots and a $(-1,0 \mathrm{j})$ point. The phase delay at $4 \mathrm{~Hz}$ to $8 \mathrm{~Hz}$ move the plots close to the $(-1,0 \mathrm{j})$ point and worsens the sensitive function. High sensitive function means that the controller cannot suppress the vibration disturbance very well at that frequency.

The conventional method uses the high-pass filter (HPF) to eliminates the effect of road slopes. This paper does not use it to make it easier to compare the LPF effect. Because HPF does not change gain nor phase over the bandwidth, there is no effect of HPF over bandwidth, such as $0.6 \mathrm{~Hz}$.

\section{Proposed Filter Design Method for tSH Con- trol Method}

4.1 General Filter Design Method We design precompensation filters based on the identified frequency response fitting data. The design procedure is described below, and its overview is shown in Fig. 9.

Step 1 Apply the tSH control method without filters to the vehicle plant.

Step 2 Lower the gain over the identified bandwidth (greater than $10 \mathrm{~Hz}$ ) using the high-cutoff-frequency LPF.

Step 3 Stabilize the gain of the identified resonance at $10 \mathrm{~Hz}$ using notch filters.
Step 4 Lead the phase of the target frequency at $4 \mathrm{~Hz}$ to $8 \mathrm{~Hz}$ to lower the sensitive function.

Step 5 Tune the tradeoff of the gain and phase based on the sensitive function.

The transfer functions of the filter components are expressed as follows:

LPF (e.g. 2nd order Butterworth LPF)

$$
D_{\mathrm{LPF}}(s)=\frac{w_{c}^{2}}{s^{2}+2 w_{c} s+w_{c}^{2}}, \cdots
$$

where $w_{c}$ is a cutoff frequency.

Notch filter:

$$
D_{\text {notch }}(s)=\frac{s^{2}+2 d \zeta w_{\mathrm{n}} s+w_{\mathrm{n}}^{2}}{s^{2}+2 \zeta w_{\mathrm{n}} s+w_{\mathrm{n}}^{2}},
$$

where $d$ is a width parameter, $\zeta$ is a depth parameter, and $w_{\max }$ is the center angular frequency.

Phase lead filter:

$$
D_{\text {lead }}(s)=\frac{T s+1}{a T s+1}, \cdots
$$

where

$$
\begin{aligned}
a & =\frac{1-\sin \phi_{\max }}{1+\sin \phi_{\max }}, \\
T & =\frac{1}{\sqrt{a} w_{\mathrm{m}}}, \cdots
\end{aligned}
$$

$\phi_{\max }$ is the maximum lead phase, and $w_{\mathrm{m}}$ is the center angular frequency.

This procedure is applicable for all wheels independently. However, the adjustment is also assumed to be needed for mutual interference between each wheel.

\subsection{Application of Filter Design for Experimental Ve-} hicle This section shows a filter design example for the experimental vehicle. Fig. 10(a) shows the Nyquist plots of the plants with the tSH control with no filters (Step 1). This case is unstable around $15 \mathrm{~Hz}$ and $43 \mathrm{~Hz}$. Because the system identification bandwidth is $10 \mathrm{~Hz}$, we lower the gain over $10 \mathrm{~Hz}$ using a high-cutoff-frequency LPF for gain stabilization and model error suppression (Step 2). In this case, we apply the second-order Butterworth LPF, whose cutoff frequency is $10 \mathrm{~Hz}$. Next, Fig. 10(b) shows the Nyquist plots of the plants with the tSH control with only a high-cutofffrequency LPF. This case is unstable because of the resonance at $9.5 \mathrm{~Hz}$, which is under the system identification bandwidth. This resonance comes from unsprung mass and tire stiffness. Therefore the resonance frequency does not change against the passenger weight change. A notch filter should stabilize this identified resonance by the gain (Step 3 ). The notch filter strength has a tradeoff between gain stability around $9.5 \mathrm{~Hz}$ and phase delay instability under $9.5 \mathrm{~Hz}$. We can now stabilize the tSH control. Finally, we design the phase lead filter to lower the sensitive function (Step 4). In this example, we lead the phase most at $4 \mathrm{~Hz}$ to $8 \mathrm{~Hz}$, which is significant for the driver's ride comfort ${ }^{(17)}$. The stronger the

$$
D(s)=\frac{63^{2}}{s^{2}+2 \times 63 s+63^{2}} \frac{s^{2}+2 \times 2.6 \times 0.19 \times 66 s+66^{2}}{s^{2}+2 \times 0.19 \times 66 s+66^{2}} \frac{0.055 s+1}{0.17 \times 0.055 s+1}
$$




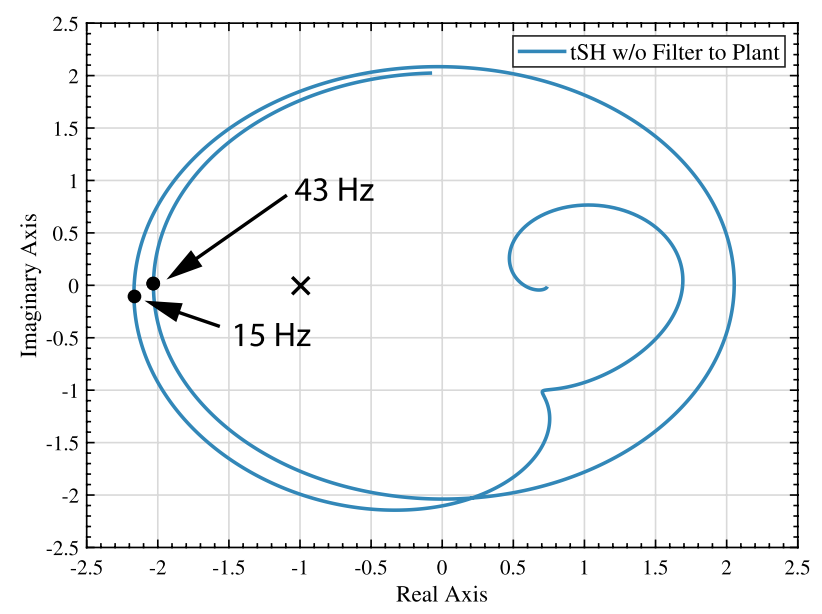

(a) Step 1: Nyquist plots of the plants with tSH control with no filter.

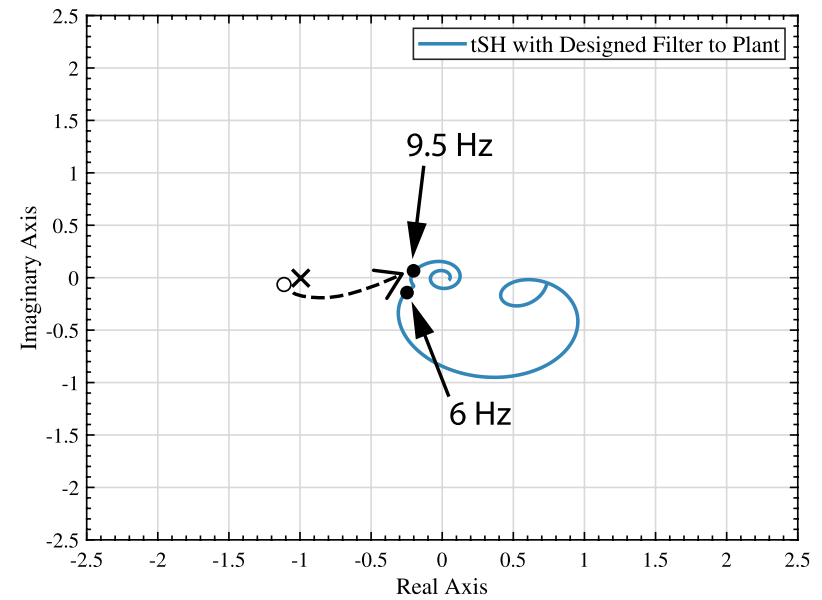

(c) Step 3: Nyquist plots of the plants with tSH control with LPF and notch filter

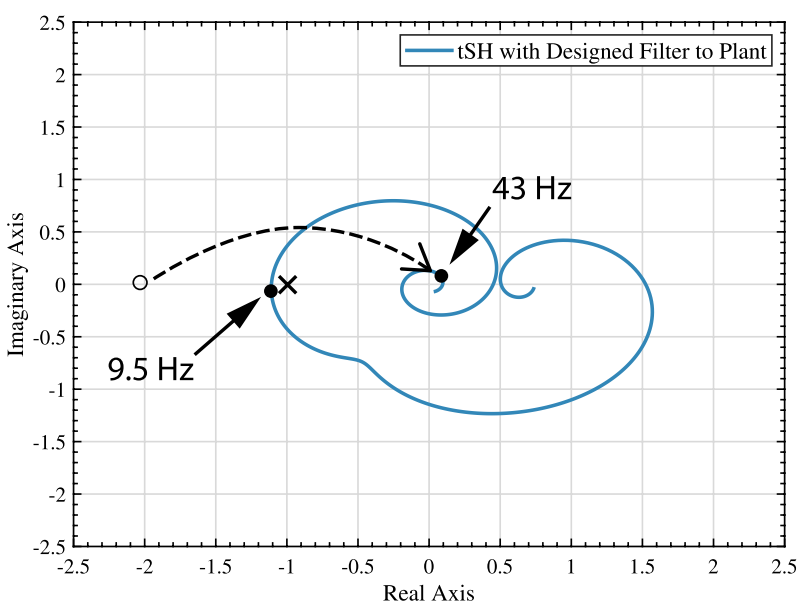

(b) Step 2: Nyquist plots of the plants with tSH control with LPF.

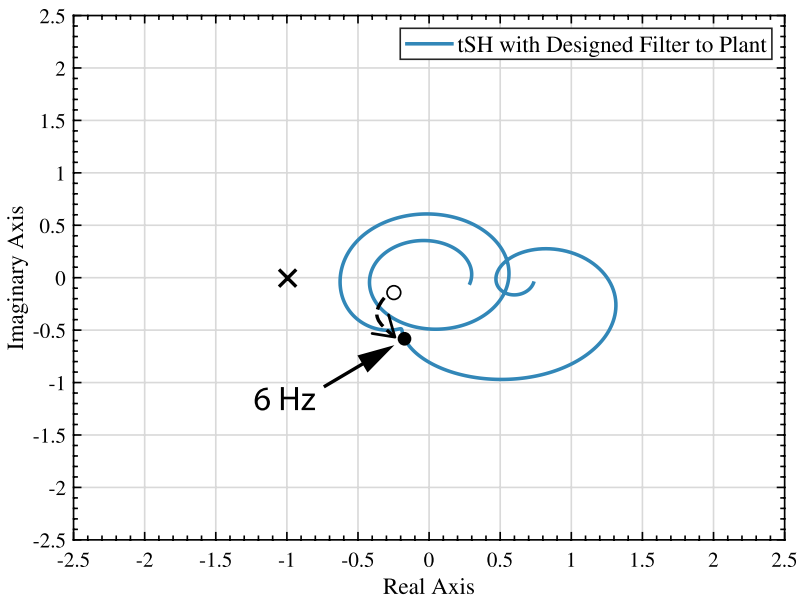

(d) Step 4, 5: Nyquist plots of the plants with tSH control with LPF, notch filter, and phase lead filter.

Fig. 10. Nyquist plots of filter design procedure

phase lead filter is, the lower the sensitive function at $4 \mathrm{~Hz}$ to $8 \mathrm{~Hz}$ is. However, It is needed to tune the parameters in a tradeoff with gain instability over the phase lead filter bandwidth. The transfer function of the designed filter is shown in (14).

The Nyquist diagram of the plants with the conventional LPF and the proposed filter is displayed in Fig. 11. The point of $6 \mathrm{~Hz}$ of the proposed filter, which is in the vibration suppression target frequency, is farther from the -1 point than the conventional LPF. This indicates the low-sensitive function at $6 \mathrm{~Hz}$, as shown in Fig. 12. There exists a tradeoff between the sensitive function of the target frequency ( $4 \mathrm{~Hz}$ to $8 \mathrm{~Hz}$ ) and other frequencies such as $35 \mathrm{~Hz}$ in this case. Thus, it is necessary to tune the sensitive function based on the desired vibration suppression frequency characteristics.

\section{Experimental Validation}

The experimental vehicle with the $\mathrm{tSH}$ control with the conventional LPF and the proposed filter was run on a road with a random surface at $60 \mathrm{~km} / \mathrm{h}$. The designed controller is applied for the rear left wheel motor. The conventional LPF is a first-order LPF with a cutoff frequency of $3 \mathrm{~Hz}$, and the proposed filter is designed as discussed in the previous section. The gain $\alpha$ of the tSH control is set to $\alpha=1.5$.

The power spectral density (PSD) of the vertical

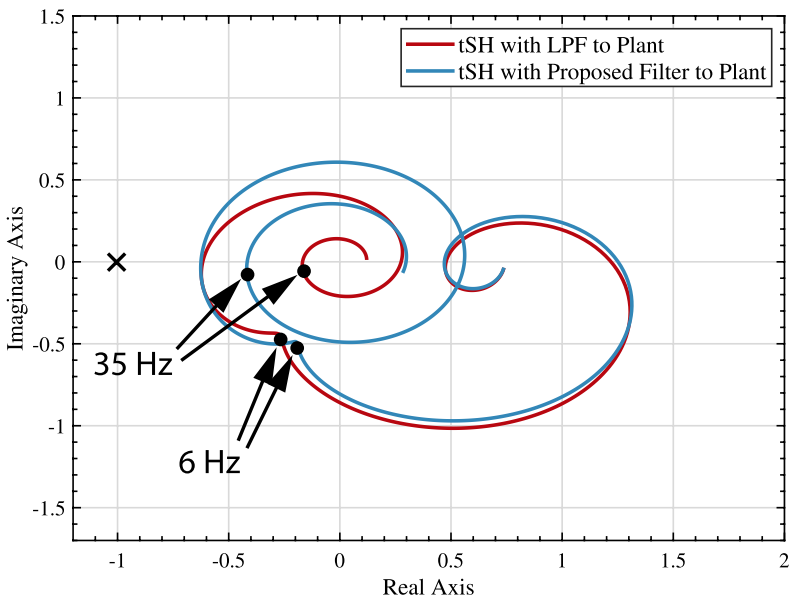

Fig. 11. Nyquist plots of plants with tSH control with filter

acceleration of the sprung mass is shown in Fig. 13. The $4 \mathrm{~Hz}$ to $8 \mathrm{~Hz}$ vibration is the most significant for drivers ${ }^{(17)}$. As in the filter design, the vibration at the target frequency $4 \mathrm{~Hz}$ to $8 \mathrm{~Hz}$ of the proposed filter is lower than that of the conventional LPF. There exists a tradeoff between $4 \mathrm{~Hz}$ to $8 \mathrm{~Hz}$ and $25 \mathrm{~Hz}$ to $30 \mathrm{~Hz}$. $25 \mathrm{~Hz}$ to $30 \mathrm{~Hz}$ vibrations are less critical than $4 \mathrm{~Hz}$ to $8 \mathrm{~Hz}$ for drivers. Although $25 \mathrm{~Hz}$ to $30 \mathrm{~Hz}$ 


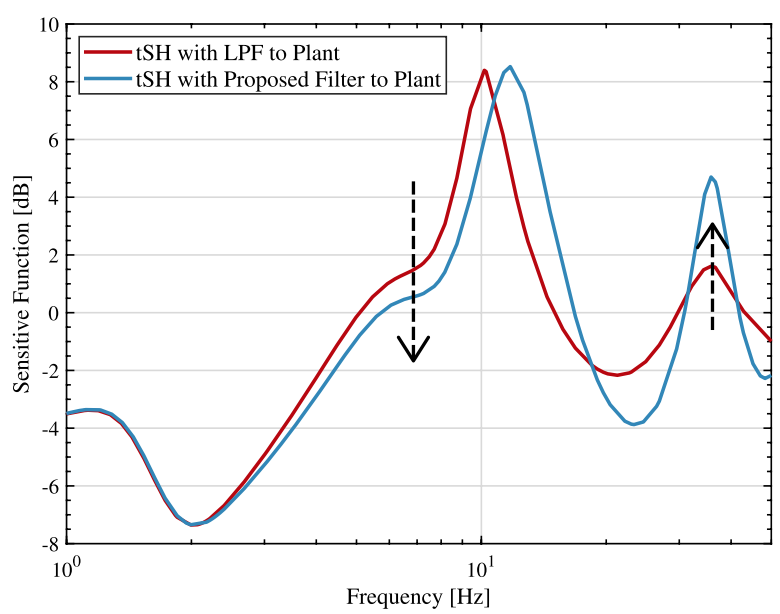

Fig. 12. Sensitive function of plants with tSH control and filter

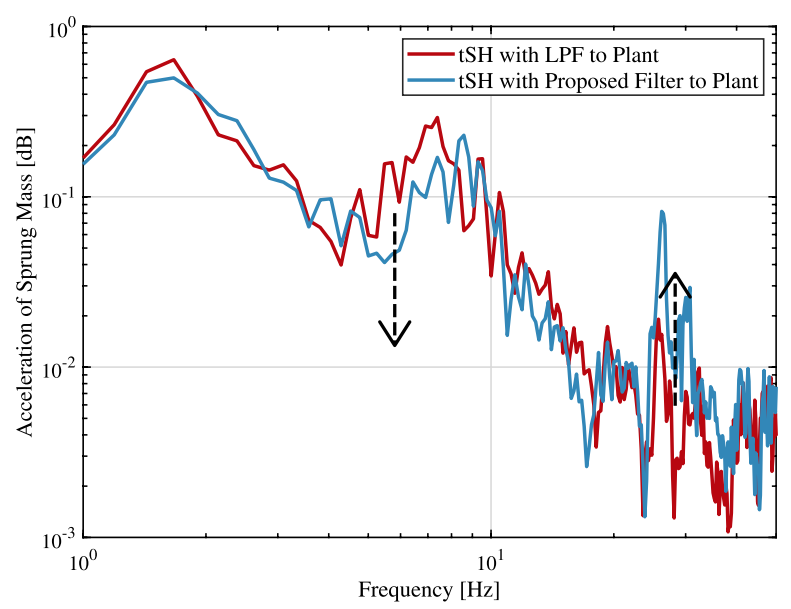

Fig. 13. PSD of acceleration of sprung mass

vibrations are also perceived as noise, the noise was not a problem in the sensory test. It may be the need to suppress the $25 \mathrm{~Hz}$ to $30 \mathrm{~Hz}$ noise in the future when other vehicle noises get more silent.

The difference of the exciting frequency around $30 \mathrm{~Hz}$ between the design process and the experimental result comes from phase modeling error. There exists phase modeling error over $10 \mathrm{~Hz}$ because a low-dimensional fitting model is used. However, the phase difference does not matter in the design process because the high bandwidth gain is lowered by the low pass filter as a whole.

The experimental results show the validation of the modelbased design of the pre-compensation filter for realizing desired frequency characteristics.

\section{Conclusion}

This paper proposed the model-based design of a precompensation filter for the $\mathrm{tSH}$ control method. The conventional $\mathrm{tSH}$ control method uses a low-cutoff-frequency LPF to stabilize the system. However, a low-cutoff-frequency LPF delays the phase at the target frequency of $4 \mathrm{~Hz}$ to $8 \mathrm{~Hz}$, which worsens the vibration suppression performance. In the proposed model-based design method, we can design a pre-compensation filter to realize the desired frequency characteristics based on the identified frequency response of the vehicle. As a result, it is possible to increase the cutoff frequency of the LPF and improve the vibration suppression performance at the target frequency. The match between the sensitive function and the experimental PSD results validates the proposed model-based filter design method. In the experimental results, the proposed method improved the vibration suppression performance at $4 \mathrm{~Hz}$ to $8 \mathrm{~Hz}$, which is most significant for driver ride comfort. In the filter design process, there exists a tradeoff between several frequencies. By identifying the driver's wide range of vibration sensitivity, we can optimize overall frequency characteristics. In this paper, the mutual interference between each wheel is not considered. The integrated filter design of all four wheels will be future work.

\section{Acknowledgment}

This work was partially supported by JSPS KAKENHI Grant Number JP18H03768.

\section{References}

(1) Y. Hori: "Future Vehicle Driven by Electricity and Control-Research on FourWheel-Motored “UOT Electric March II"”, IEEE Transactions on Industrial Electronics, Vol.51, No.5, pp.954-962 (2004)

( 2 ) V.-D. Doan, H. Fujimoto, T. Koseki, T. Yasuda, H. Kishi, and T. Fujita: "Iterative Dynamic Programming for Optimal Control Problem with Isoperimetric Constraint and Its Application to Optimal Eco-driving Control of Electric Vehicle", IEEJ Journal of Industry Applications, Vol.7, No.1, pp.80-92 (2018)

( 3 ) T. Ohhira and A. Shimada: "Movement Control Based on Model Predictive Control with Disturbance Suppression using Kalman Filter including Disturbance Estimation”, IEEJ Journal of Industry Applications, Vol.7, No.5, pp.387-395 (2018)

( 4 ) S. Murata: "Innovation by in-wheel-motor drive unit", Vehicle System Dynamics, Vol.50, No.6, pp.807-830 (2012)

( 5 ) V. Ivanov, D. Savitski, J. Orus, J.M.R. Fortun, A. Sorniotti, and P. Gruber: "All-wheel-drive electric vehicle with on-board motors: Experimental validation of the motion control systems", IECON 2015 - 41st Annual Conference of the IEEE Industrial Electronics Society, pp.1729-1734 (2015)

( 6 ) J. M. Rodriguez, R. Meneses, and J. Orus: "Active vibration control for electric vehicle compliant drivetrains", IECON 2013 - 39th Annual Conference of the IEEE Industrial Electronics Society, pp.2590-2595 (2013)

( 7 ) E. Katsuyama: "Decoupled 3D moment control for vehicle motion using InWheel motors", SAE International Journal of Passenger Cars - Mechanical Systems, Vol.6, pp.137-146 (2013)

( 8 ) N. Ochi, H. Fujimoto, and Y. Hori: "Proposal of roll angle control method using positive and negative anti-dive force for electric vehicle with four inwheel motors", 2013 IEEE International Conference on Mechatronics, ICM 2013, pp.816-821 (2013)

( 9 ) M. Liu, F. Gu, and Y. Zhang: "Ride comfort optimization of in-wheelmotor electric vehicles with in-wheel vibration absorbers", Energies, Vol.10, No.1647, pp.1-21 (2017)

(10) V. Roel, B. Igo, and H. Nijmeijer: "Influence of in-wheel motors on the ride comfort of electric vehicles", Int. Symposium on Advanced Vehicle Control (AVEC), pp.835-840 (2010)

(11) A. Martyn and H. Damian: "Unsprung mass with in-wheel motors-myths and realities", Int. Symposium on Advanced Vehicle Control (AVEC), pp.261-266 (2010)

(12) E. Katsuyama and A. Omae: "Improvement of Ride Comfort by Unsprung Negative Skyhook Damper Control Using In-Wheel Motors", SAE International Journal of Alternative Powertrains, Vol.5, No.1, pp.2016-01-1678 (2016)

(13) S. Ohno and K. Ito: "Unsprung vibration control for in-wheel-motor EV", JSAE Autumn Congress 2014, No.103, pp.21-24 (2014)

(14) H. Fukudome: "Reduction of longitudinal vehicle vibration using InWheel motors", SAE Technical Paper Series (2016)

(15) A. Takesaki, M. Kawafuku, M. Iwasaki, and T. Fujii: "Vibration Suppression Control System for Electric Vehicle Considering Longitudinal Vibration”, Proc. of IEEJ Technical Meeting Record, Vol.97, pp.7-12 (2010)

(16) E. Katsuyama: "Improvement of ride comfort by triple skyhook control", in 9th International Munich Chassis Symposium 2018, pp.215-234 (2018)

(17) "ISO Standard 2631-1: Mechanical vibration and shock-Evaluation of 
human exposure to whole-body vibration Part 1" (1997)

(18) R. Pintelon, P. Guillaume, Y. Rolain, J. Schoukens, and H. Van Hamme: "Parametric identification of transfer functions in the frequency domain-a survey," IEEE Transactions on Automatic Control, Vol.39, No.11, pp.22452260 (1994)

(19) M.C. Smith: "Synthesis of mechanical networks: the inerter", IEEE Transactions on Automatic Control, Vol.47, No.10, pp.1648-1662 (2002)

Tomonori Suzuki (Student Member) received the B.S. degree from

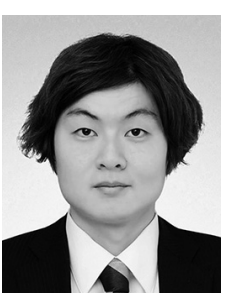
The University of Tokyo in 2019. He is currently working towards an M.S. degree in the Department of Advanced Energy, Graduate School of Frontier Sciences, the University of Tokyo. His interests are in control engineering, motion control, and vibration suppression control. He is a student member of the Institute of Electrical and Electronics Engineers and the Institute of Electrical Engineers of Japan.

Masahiro Mae (Student Member) received the B.E. and M.S. degree

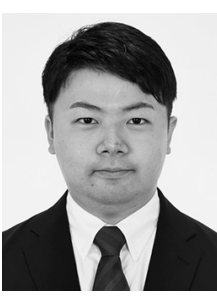
from The University of Tokyo in 2018 and 2020, respectively. He is currently working towards the Ph.D. degree in the Department of Electrical Engineering and Information Systems, Graduate School of Engineering, The University of Tokyo. His research interests are in control engineering, precision motion control, multirate control and multi-input multi-output systems. He is a student member of the Institute of Electrical and Electronics Engineers.

Takuma Takeuchi (Non-member) received M.S. degree in The Uni-

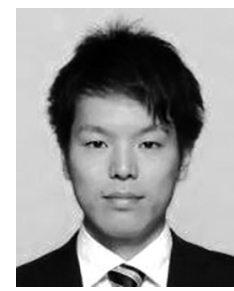
versity of Tokyo, Japan in 2017. In 2017, He joined Toyota Motor Co., Ltd., Japan. His research interest includes vehicle motion control.
Hiroshi Fujimoto (Senior Member) received the Ph.D. degree in the

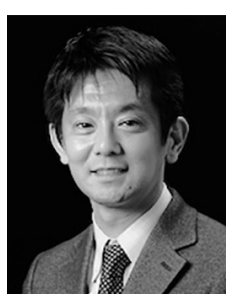
Department of Electrical Engineering from the University of Tokyo in 2001. In 2001, he joined the Department of Electrical Engineering, Nagaoka University of Technology, Niigata, Japan, as a research associate. From 2002 to 2003, he was a visiting scholar in the School of Mechanical Engineering, Purdue University, U.S.A. In 2004, he joined the Department of Electrical and Computer Engineering, Yokohama National University, Yokohama, Japan, as a lecturer and he became an associate professor in 2005 . He is currently an associate professor of the University of Tokyo since 2010. He received the Best Paper Awards from the IEEE Transactions on Industrial Electronics in 2001 and 2013, Isao Takahashi Power Electronics Award in 2010, Best Author Prize of SICE in 2010, the Nagamori Grand Award in 2016, and First Prize Paper Award IEEE Transactions on Power Electronics in 2016. His interests are in control engineering, motion control, nano-scale servo systems, electric vehicle control, motor drive, visual servoing, and wireless motors. $\mathrm{He}$ is a senior member of IEE of Japan and IEEE. He is also a member of the Society of Instrument and Control Engineers, the Robotics Society of Japan, and the Society of Automotive Engineers of Japan. He is an associate editor of IEEE/ASME Transactions on Mechatronics from 2010 to 2014, IEEE Industrial Electronics Magazine from 2006, IEE of Japan Transactions on Industrial Application from 2013, and Transactions on SICE from 2013 to 2016. He is a chairperson of JSAE vehicle electrification committee from 2014 and a past chairperson of IEEE/IES Technical Committee on Motion Control from 2012 to 2013.

Etsuo Katsuyama (Non-member) received the B.S. degree in Tokyo

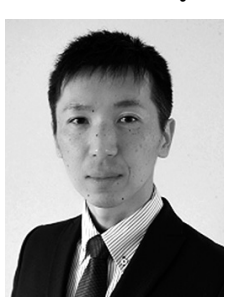
Institute of Technology in 1997. He joined Unisia Jecs in Japan in 1997. From 2007 to 2010, he joined Toyota Motorsport GmbH in Germany as a chassis engineer of formula 1 . He currently joins Toyota Motor Corporation in Japan as a chassis engineer of passenger vehicles. He received the Arch T. Colwell Merit Award from the Society of Automotive Engineers in 2015, and the Outstanding Technical Paper Awards from the Society of Automotive Engineers of Japan in 2013 and 2018. 